Pacific

Journal of

Mathematics

THE ASYMPTOTIC BEHAVIOR OF PALAIS-SMALE SEQUENCES ON MANIFOLDS WITH BOUNDARY

SÉRGIO AlmaraZ

Volume 269 No. 1

May 2014 


\title{
THE ASYMPTOTIC BEHAVIOR OF PALAIS-SMALE SEQUENCES ON MANIFOLDS WITH BOUNDARY
}

\author{
SÉRgio AlMARAZ
}

We describe the asymptotic behavior of Palais-Smale sequences associated to certain Yamabe-type equations on manifolds with boundary. We prove that each of those sequences converges to a solution of the limit equation plus a finite number of "bubbles" which are obtained by rescaling fundamental solutions of the corresponding Euclidean equations.

\section{Introduction}

Let $\left(M^{n}, g\right)$ be a compact Riemannian manifold with boundary $\partial M$ and dimension $n \geq 3$. For $u \in H^{1}(M)$, we consider the following family of equations, indexed by $v \in \mathbb{N}$ :

$$
\begin{cases}\Delta_{g} u=0 & \text { in } M, \\ \frac{\partial}{\partial \eta_{g}} u-h_{v} u+u^{\frac{n}{n-2}}=0 & \text { on } \partial M,\end{cases}
$$

and their associated functionals

$$
I_{g}^{v}(u)=\frac{1}{2} \int_{M}|d u|_{g}^{2} d v_{g}+\frac{1}{2} \int_{\partial M} h_{\nu} u^{2} d \sigma_{g}-\frac{n-2}{2(n-1)} \int_{\partial M}|u|^{\frac{2(n-1)}{n-2}} d \sigma_{g} .
$$

Here, $\left\{h_{v}\right\}_{v \in \mathbb{N}}$ is a sequence of functions in $C^{\infty}(\partial M), \Delta_{g}$ is the Laplace-Beltrami operator, and $\eta_{g}$ is the inward unit normal vector to $\partial M$. Moreover, $d v_{g}$ and $d \sigma_{g}$ are the volume forms of $M$ and $\partial M$ respectively and $H^{1}(M)$ is the Sobolev space $H^{1}(M)=\left\{u \in L^{2}(M): d u \in L^{2}(M)\right\}$.

Definition 1.1. We say that $\left\{u_{v}\right\}_{v \in \mathbb{N}} \subset H^{1}(M)$ is a Palais-Smale sequence for $\left\{I_{g}^{v}\right\}$ if

(i) $\left\{I_{g}^{\nu}\left(u_{v}\right)\right\}_{v \in \mathbb{N}}$ is bounded, and

(ii) $d I_{g}^{v}\left(u_{v}\right) \rightarrow 0$ strongly in $H^{1}(M)^{\prime}$ as $v \rightarrow \infty$.

Supported by CAPES and FAPERJ (Brazil). MSC2010: primary 35J65; secondary 53C21.

Keywords: Riemannian manifold, Palais-Smale sequence, manifold with boundary, blow-up. 
In this paper we establish a result describing the asymptotic behavior of those Palais-Smale sequences. This work is inspired by Struwe's theorem [1984] for equations $\Delta u+\lambda u+|u|^{4 /(n-2)} u=0$ on Euclidean domains. We refer the reader to [Druet et al. 2004, Chapter 3] for a version of Struwe's theorem on closed Riemannian manifolds, and to [Cao et al. 2001; Chabrowski and Girão 2002; Pierotti and Terracini 1995] for similar equations with boundary conditions.

Roughly speaking, as $v \rightarrow \infty$ and $h_{v} \rightarrow h_{\infty}$, we prove that each Palais-Smale sequence $\left\{u_{v} \geq 0\right\}_{v \in \mathbb{N}}$ is $H^{1}(M)$-asymptotic to a nonnegative solution of the limit equations

$$
\begin{cases}\Delta_{g} u=0 & \text { in } M, \\ \frac{\partial}{\partial \eta_{g}} u-h_{\infty} u+u^{\frac{n}{n-2}}=0 & \text { on } \partial M,\end{cases}
$$

plus a finite number of "bubbles" obtained by rescaling fundamental positive solutions of the Euclidean equations

$$
\begin{cases}\Delta u=0 & \text { in } \mathbb{R}_{+}^{n}, \\ \frac{\partial}{\partial y_{n}} u+u^{\frac{n}{n-2}}=0 & \text { on } \partial \mathbb{R}_{+}^{n},\end{cases}
$$

where $\mathbb{R}_{+}^{n}=\left\{\left(y_{1}, \ldots, y_{n}\right) \in \mathbb{R}^{n}: y_{n} \geq 0\right\}$.

Palais-Smale sequences frequently appear in the blow-up analysis of geometric problems. In the particular case when $h_{\infty}$ is $(n-2) / 2$ times the boundary mean curvature, the equations (1-3) are satisfied by a positive smooth function $u$ representing a conformal scalar-flat Riemannian metric $u^{4 /(n-2)} g$ with positive constant boundary mean curvature. The existence of those metrics is the Yamabe-type problem for manifolds with boundary introduced in [Escobar 1992].

An application of our result is the blow-up analysis performed in [Almaraz 2012] for the proof of a convergence theorem for a Yamabe-type flow introduced in [Brendle 2002].

We now begin to state our theorem more precisely.

Convention. We assume that there is some $h_{\infty} \in C^{\infty}(\partial M)$ and $C>0$ such that $h_{v} \rightarrow h_{\infty}$ in $L^{2}(\partial M)$ as $v \rightarrow \infty$ and $\left|h_{v}(x)\right| \leq C$ for all $x \in \partial M, v \in \mathbb{N}$. This obviously implies that $h_{v} \rightarrow h_{\infty}$ in $L^{p}(\partial M)$ as $v \rightarrow \infty$, for any $p \geq 1$.

Notation. If $(M, g)$ is a Riemannian manifold with boundary $\partial M$, we will denote by $D_{r}(x)$ the metric ball in $\partial M$ with center at $x \in \partial M$ and radius $r$.

If $z_{0} \in \mathbb{R}_{+}^{n}$, we set $B_{r}^{+}\left(z_{0}\right)=\left\{z \in \mathbb{R}_{+}^{n}:\left|z-z_{0}\right|<r\right\}$. We define

$$
\partial^{+} B_{r}^{+}\left(z_{0}\right)=\partial B_{r}^{+}\left(z_{0}\right) \cap \mathbb{R}_{+}^{n}, \quad \text { and } \quad \partial^{\prime} B_{r}^{+}\left(z_{0}\right)=B_{r}^{+}\left(z_{0}\right) \cap \partial \mathbb{R}_{+}^{n} .
$$

Thus, $\partial^{\prime} B_{r}^{+}\left(z_{0}\right)=\varnothing$ if $z_{0}=\left(z_{0}^{1}, \ldots, z_{0}^{n}\right)$ satisfies $z_{0}^{n}>r$. 
We define the Sobolev space $D^{1}\left(\mathbb{R}_{+}^{n}\right)$ as the completion of $C_{0}^{\infty}\left(\mathbb{R}_{+}^{n}\right)$ with respect to the norm

$$
\|u\|_{D^{1}\left(\mathbb{R}_{+}^{n}\right)}=\sqrt{\int_{\mathbb{R}_{+}^{n}}|d u(y)|^{2} d y .}
$$

It follows from a Liouville-type theorem established in [Li and Zhu 1995] (see also [Escobar 1990] and [Chipot et al. 1996]) that any nonnegative solution in $D^{1}\left(\mathbb{R}_{+}^{n}\right)$ to the equations $(1-4)$ is of the form

$$
U_{\epsilon, a}(y)=\left(\frac{\epsilon}{\left(y_{n}+\epsilon /(n-2)\right)^{2}+|\bar{y}-a|^{2}}\right)^{\frac{n-2}{2}}, \quad a \in \mathbb{R}^{n-1}, \epsilon>0,
$$

or is identically zero; see Remark 2.5. By [Escobar 1988] or [Beckner 1993] we have the sharp Euclidean Sobolev inequality

$$
\left(\int_{\partial \mathbb{R}_{+}^{n}}|u(y)|^{\frac{2(n-1)}{n-2}} d y\right)^{\frac{n-2}{n-1}} \leq K_{n}^{2} \int_{\mathbb{R}_{+}^{n}}|d u(y)|^{2} d y,
$$

for $u \in D^{1}\left(\mathbb{R}_{+}^{n}\right)$, which has the family of functions (1-5) as extremal functions. Here,

$$
K_{n}=\left(\frac{n-2}{2}\right)^{-1 / 2} \sigma_{n-1}^{-\frac{1}{2(n-1)}},
$$

where $\sigma_{n-1}$ is the area of the unit $(n-1)$-sphere in $\mathbb{R}^{n}$. Up to a multiplicative constant, the functions defined by (1-5) are the only nontrivial extremal ones for the inequality (1-6).

Definition 1.2. Fix $x_{0} \in \partial M$ and geodesic normal coordinates for $\partial M$ centered at $x_{0}$. Let $\left(x_{1}, \ldots, x_{n-1}\right)$ be the coordinates of $x \in \partial M$ and $\eta_{g}(x)$ be the inward unit vector normal to $\partial M$ at $x$. For small $x_{n} \geq 0$, the point $\exp _{x}\left(x_{n} \eta_{g}(x)\right) \in M$ is said to have Fermi coordinates $\left(x_{1}, \ldots, x_{n}\right)$ (centered at $\left.x_{0}\right)$.

For small $\rho>0$ the Fermi coordinates centered at $x_{0} \in \partial M$ define a smooth map $\psi_{x_{0}}: B_{\rho}^{+}(0) \subset \mathbb{R}_{+}^{n} \rightarrow M$.

We define the functional $I_{g}^{\infty}$ by the same expression as $I_{g}^{v}$, with $h_{v}=h_{\infty}$ for all $v$, and state our main theorem as follows:

Theorem 1.3. Let $\left(M^{n}, g\right)$ be a compact Riemannian manifold with boundary $\partial M$ and dimension $n \geq 3$. Suppose $\left\{u_{v} \geq 0\right\}_{v \in \mathbb{N}}$ is a Palais-Smale sequence for $\left\{I_{g}^{v}\right\}$. Then there exist $m \in\{0,1,2, \ldots\}$, a nonnegative solution $u^{0} \in H^{1}(M)$ of (1-3), and $m$ nontrivial nonnegative solutions $U^{j}=U_{\epsilon_{j}, a_{j}} \in D^{1}\left(\mathbb{R}_{+}^{n}\right)$ of (1-4), sequences $\left\{R_{v}^{j}>0\right\}_{v \in \mathbb{N}}$, and sequences $\left\{x_{v}^{j}\right\}_{v \in \mathbb{N}} \subset \partial M, 1 \leq j \leq m$, the whole satisfying the following conditions for $1 \leq j \leq m$, possibly after taking subsequences:

(i) $R_{v}^{j} \rightarrow \infty$ as $v \rightarrow \infty$.

(ii) $x_{v}^{j}$ converges as $v \rightarrow \infty$. 
(iii) $\left\|u_{v}-u^{0}-\sum_{j=1}^{m} \eta_{\nu}^{j} u_{v}^{j}\right\|_{H^{1}(M)} \rightarrow 0$ as $v \rightarrow \infty$, where

$$
u_{v}^{j}(x)=\left(R_{v}^{j}\right)^{(n-2) / 2} U^{j}\left(R_{v}^{j} \psi_{x_{v}^{j}}^{-1}(x)\right) \quad \text { for } x \in \psi_{x_{v}^{j}}\left(B_{2 r_{0}}^{+}(0)\right) .
$$

Here, $r_{0}>0$ is small, the

$$
\psi_{x_{v}^{j}}: B_{2 r_{0}}^{+}(0) \subset \mathbb{R}_{+}^{n} \rightarrow M
$$

are Fermi coordinates centered at $x_{v}^{j} \in \partial M$, and the $\eta_{v}^{j}$ are smooth cutoff functions such that $\eta_{v}^{j} \equiv 1$ in $\psi_{x_{v}^{j}}\left(B_{r_{0}}^{+}(0)\right)$ and $\eta_{v}^{j} \equiv 0$ in $M \backslash \psi_{x_{v}^{j}}\left(B_{2 r_{0}}^{+}(0)\right)$.

Moreover,

$$
I_{g}^{v}\left(u_{v}\right)-I_{g}^{\infty}\left(u^{0}\right)-\frac{m}{2(n-1)} K_{n}^{-2(n-1)} \rightarrow 0 \quad \text { as } v \rightarrow \infty,
$$

and we can assume that for all $i \neq j$

$$
\frac{R_{v}^{i}}{R_{v}^{j}}+\frac{R_{v}^{j}}{R_{v}^{i}}+R_{v}^{i} R_{v}^{j} d_{g}\left(x_{v}^{i}, x_{v}^{j}\right)^{2} \rightarrow \infty \quad \text { as } v \rightarrow \infty .
$$

Remark 1.4. Relations of the type (1-7) were previously obtained in [Bahri and Coron 1988; Brezis and Coron 1985].

\section{Proof of the main theorem}

The rest of this paper is devoted to the proof of Theorem 1.3, which will be carried out in several lemmas. Our presentation will follow the same steps as Chapter 3 of [Druet et al. 2004], with the necessary modifications.

Lemma 2.1. Let $\left\{u_{v}\right\}$ be a Palais-Smale sequence for $\left\{I_{g}^{\nu}\right\}$. Then there exists $C>0$ such that $\left\|u_{v}\right\|_{H^{1}(M)} \leq C$ for all $v$.

Proof. It suffices to prove that $\left\|d u_{v}\right\|_{L^{2}(M)}$ and $\left\|u_{v}\right\|_{L^{2}(\partial M)}$ are uniformly bounded. The proof follows the same arguments as [Druet et al. 2004, p. 27].

Define $I_{g}$ as the functional in (1-2) when $h_{v} \equiv 0$ for all $v$.

Lemma 2.2. Let $\left\{u_{v} \geq 0\right\}$ be a Palais-Smale sequence for $\left\{I_{g}^{v}\right\}$ such that $u_{v} \rightarrow$ $u^{0} \geq 0$ in $H^{1}(M)$, and set $\hat{u}_{v}=u_{v}-u^{0}$. Then $\left\{\hat{u}_{v}\right\}$ is a Palais-Smale sequence for $\left\{I_{g}\right\}$ and satisfies

$$
I_{g}\left(\hat{u}_{v}\right)-I_{g}^{v}\left(u_{v}\right)+I_{g}^{\infty}\left(u^{0}\right) \rightarrow 0 \quad \text { as } v \rightarrow \infty .
$$

Moreover, $u^{0}$ is a (weak) solution of (1-3).

Proof. First, observe that $u_{v} \rightarrow u^{0}$ in $H^{1}(M)$ implies that $u_{v} \rightarrow u^{0}$ in $L^{\frac{n}{n-2}}(\partial M)$ and a.e. in $\partial M$. Using the facts that $d I_{g}^{v}\left(u_{v}\right) \phi \rightarrow 0$ for any $\phi \in C^{\infty}(\bar{M})$ and $h_{v} \rightarrow h_{\infty}$ in $L^{p}(\partial M)$ for any $p \geq 1$, it is not difficult to see that the last assertion of Lemma 2.2 follows. 
In order to prove (2-1), we first observe that

$$
I_{g}^{v}\left(u_{v}\right)=I_{g}\left(\hat{u}_{v}\right)+I_{g}^{\infty}\left(u^{0}\right)-\frac{(n-2)}{2(n-1)} \int_{\partial M} \Phi_{\nu} d \sigma_{g}+o(1),
$$

where $\Phi_{v}=\left|\hat{u}_{v}+u^{0}\right|^{\frac{2(n-1)}{n-2}}-\left|\hat{u}_{v}\right|^{\frac{2(n-1)}{n-2}}-\left|u^{0}\right|^{\frac{2(n-1)}{n-2}}$ and $o(1) \rightarrow 0$ as $v \rightarrow \infty$. Then (2-1) follows from the fact that there exists $C>0$ such that

$$
\int_{\partial M} \Phi_{\nu} d \sigma_{g} \leq C \int_{\partial M}\left|\hat{u}_{\nu}\right|^{\frac{n}{n-2}}\left|u^{0}\right| d \sigma_{g}+C \int_{\partial M}\left|u^{0}\right|^{\frac{n}{n-2}}\left|\hat{u}_{\nu}\right| d \sigma_{g} \quad \text { for all } v,
$$

and, by basic integration theory, the right side of this last inequality goes to 0 as $v \rightarrow \infty$.

Now we prove that $\left\{\hat{u}_{v}\right\}$ is a Palais-Smale sequence for $I_{g}$. Let $\phi \in C^{\infty}(M)$. Observe that

$$
\begin{aligned}
\left|\int_{\partial M} h_{\nu} u_{\nu} \phi d \sigma_{g}-\int_{\partial M} h_{\infty} u_{\nu} \phi d \sigma_{g}\right| & \\
& \leq\left\|u_{\nu}\right\|_{L^{2}(\partial M)}\left\|h_{\nu}-h_{\infty}\right\|_{L^{2(n-1)}(\partial M)}\|\phi\|_{L^{\frac{2(n-1)}{n-2}(\partial M)}}
\end{aligned}
$$

by Hölder's inequality. Then, by the Sobolev embedding theorem,

$$
\int_{\partial M} h_{\nu} u_{\nu} \phi d \sigma_{g}=\int_{\partial M} h_{\infty} u^{0} \phi d \sigma_{g}+o\left(\|\phi\|_{H^{1}(M)}\right),
$$

from which follows that

$$
d I_{g}^{v}\left(u_{v}\right) \phi=d I_{g}\left(\hat{u}_{v}\right) \phi-\int_{\partial M} \psi_{\nu} \phi d \sigma_{g}+o\left(\|\phi\|_{H^{1}(M)}\right),
$$

where $\psi_{v}=\left|\hat{u}_{v}+u^{0}\right|^{\frac{2}{n-2}}\left(\hat{u}_{v}+u^{0}\right)-\left|\hat{u}_{v}\right|^{\frac{2}{n-2}} \hat{u}_{v}-\left|u^{0}\right|^{\frac{2}{n-2}} u^{0}$.

Next we observe that there exists $C>0$ such that

$$
\int_{\partial M}\left|\psi_{\nu} \phi\right| d \sigma_{g} \leq C \int_{\partial M}\left|\hat{u}_{\nu}\right|^{\frac{2}{n-2}}\left|u^{0}\right||\phi| d \sigma_{g}+C \int_{\partial M}\left|u^{0}\right|^{\frac{2}{n-2}}\left|\hat{u}_{\nu}\right||\phi| d \sigma_{g}
$$

for all $v$, and use Hölder's inequality and basic integration theory to obtain

$$
\begin{aligned}
\int_{\partial M}\left|\psi_{\nu} \phi\right| d \sigma_{g} \\
\quad \leq\left(\left\|\left|\hat{u}_{v}\right|^{\frac{2}{n-2}} u^{0}\right\|_{L^{\frac{2(n-1)}{n}}(\partial M)}+\left\|\left|u^{0}\right|^{\frac{2}{n-2}} \hat{u}_{\nu}\right\|_{L^{\frac{2(n-1)}{n}(\partial M)}}\right)\|\phi\|_{L^{\frac{2(n-1)}{n-2}}(\partial M)} \\
\quad=o\left(\|\phi\|_{L^{\frac{2(n-1)}{n-2}}(\partial M)}\right) .
\end{aligned}
$$

We can use this and the Sobolev embedding theorem in (2-2) to conclude that

$$
d I_{g}^{v}\left(u_{v}\right) \phi=d I_{g}\left(\hat{u}_{v}\right) \phi+o\left(\|\phi\|_{H^{1}(M)}\right),
$$

finishing the proof. 
Lemma 2.3. Let $\left\{\hat{u}_{v}\right\}_{v \in \mathbb{N}}$ be a Palais-Smale sequence for $I_{g}$ such that $\hat{u}_{v} \rightarrow 0$ in $H^{1}(M)$ and $I_{g}\left(\hat{u}_{v}\right) \rightarrow \beta$ as $v \rightarrow \infty$ for some $\beta<K_{n}^{-2(n-1)} /(2(n-1))$. Then $\hat{u}_{v} \rightarrow 0$ in $H^{1}(M)$ as $v \rightarrow \infty$.

Proof. Since

$$
\int_{M}\left|d \hat{u}_{v}\right|^{2} d v_{g}-\int_{\partial M}\left|\hat{u}_{v}\right|^{\frac{2(n-1)}{n-2}} d \sigma_{g}=d I_{g}\left(\hat{u}_{v}\right) \cdot \hat{u}_{v}=o\left(\left\|\hat{u}_{v}\right\|_{H^{1}(M)}\right)
$$

and $\left\{\left\|\hat{u}_{v}\right\|_{H^{1}(M)}\right\}$ is uniformly bounded due to Lemma 2.1, we can see that

$$
\begin{aligned}
\beta+o(1)=I_{g}\left(\hat{u}_{v}\right) & =\frac{1}{2(n-1)} \int_{\partial M}\left|\hat{u}_{v}\right|^{\frac{2(n-1)}{n-2}} d \sigma_{g}+o(1) \\
& =\frac{1}{2(n-1)} \int_{M}\left|d \hat{u}_{v}\right|_{g}^{2} d v_{g}+o(1),
\end{aligned}
$$

which already implies $\beta \geq 0$. At the same time, as proved in [Li and Zhu 1997], there exists $B=B(M, g)>0$ such that

$$
\left(\int_{\partial M}\left|\hat{u}_{\nu}\right|^{\frac{2(n-1)}{n-2}} d \sigma_{g}\right)^{\frac{n-2}{n-1}} \leq K_{n}^{2} \int_{M}\left|d \hat{u}_{\nu}\right|_{g}^{2} d v_{g}+B \int_{\partial M}\left|\hat{u}_{\nu}\right|^{2} d \sigma_{g} .
$$

Since $H^{1}(M)$ is compactly embedded in $L^{2}(\partial M)$, we have $\left\|\hat{u}_{v}\right\|_{L^{2}(\partial M)} \rightarrow 0$. Then

$$
(2(n-1) \beta+o(1))^{\frac{n-2}{n-1}} \leq 2(n-1) K_{n}^{2} \beta+o(1),
$$

from which we conclude that either

$$
\frac{K_{n}^{-2(n-1)}}{2(n-1)} \leq \beta+o(1)
$$

or $\beta=0$. Hence, our hypotheses imply $\beta=0$. Using (2-3) finishes the proof.

Define the functional

$$
E(u)=\frac{1}{2} \int_{\mathbb{R}_{+}^{n}}|d u(y)|^{2} d y-\frac{n-2}{2(n-1)} \int_{\partial \mathbb{R}_{+}^{n}}|u(y)|^{\frac{2(n-1)}{n-2}} d y
$$

for $u \in D^{1}\left(\mathbb{R}_{+}^{n}\right)$ and observe that $E\left(U_{\epsilon, a}\right)=\frac{K_{n}^{-2(n-1)}}{2(n-1)}$ for any $a \in \mathbb{R}^{n-1}, \epsilon>0$.

Lemma 2.4. Let $\left\{\hat{u}_{v}\right\}_{v \in \mathbb{N}}$ be a Palais-Smale sequence for $I_{g}$. Suppose $\hat{u}_{v} \rightarrow 0$ in $H^{1}(M)$, but not strongly. Then there exist a sequence $\left\{R_{v}>0\right\}_{v \in \mathbb{N}}$ with $R_{v} \rightarrow \infty$, a convergent sequence $\left\{x_{v}\right\}_{v \in \mathbb{N}} \subset \partial M$, and a nontrivial solution $u \in D^{1}\left(\mathbb{R}_{+}^{n}\right)$ of

$$
\begin{cases}\Delta u=0 & \text { in } \mathbb{R}_{+}^{n}, \\ \frac{\partial}{\partial y_{n}} u-|u|^{2 /(n-2)} u=0 & \text { on } \partial \mathbb{R}_{+}^{n},\end{cases}
$$


the whole such that, up to a subsequence, the following holds: If

$$
\hat{v}_{\nu}(x)=\hat{u}_{v}(x)-\eta_{v}(x) R_{v}^{\frac{n-2}{2}} u\left(R_{v} \psi_{x_{v}}^{-1}(x)\right),
$$

then $\left\{\hat{v}_{v}\right\}_{v \in \mathbb{N}}$ is a Palais-Smale sequence for $I_{g}$ satisfying $\hat{v}_{v} \rightarrow 0$ in $H^{1}(M)$ and

$$
\lim _{\nu \rightarrow \infty}\left(I_{g}\left(\hat{u}_{v}\right)-I_{g}\left(\hat{v}_{v}\right)\right)=E(u) .
$$

Here, the $\psi_{x_{v}}: B_{2 r_{0}}^{+}(0) \subset \mathbb{R}_{+}^{n} \rightarrow M$ are Fermi coordinates centered at $x_{\nu}$ and the $\eta_{\nu}(x)$ are smooth cutoff functions such that $\eta_{\nu} \equiv 1$ in $\psi_{x_{v}}\left(B_{r_{0}}^{+}(0)\right)$ and $\eta_{\nu} \equiv 0$ in $M \backslash \psi_{x_{v}}\left(B_{2 r_{0}}^{+}(0)\right)$.

Proof. By the density of $C^{\infty}(M)$ in $H^{1}(M)$ we can assume that $\hat{u}_{v} \in C^{\infty}(M)$. We can also assume that $I_{g}\left(\hat{u}_{v}\right) \rightarrow \beta$ as $v \rightarrow \infty$ and, since $d I_{g}\left(\hat{u}_{v}\right) \rightarrow 0$ in $H^{1}(M)^{\prime}$, we obtain

$$
\lim _{\nu \rightarrow \infty} \int_{\partial M}\left|\hat{u}_{\nu}\right|^{\frac{2(n-1)}{n-2}} d \sigma_{g}=2(n-1) \beta \geq K_{n}^{-2(n-1)},
$$

as in the proof of Lemma 2.3. Hence, given $t_{0}>0$ small we can choose $x_{0} \in \partial M$ and $\lambda_{0}>0$ such that

$$
\int_{D_{t_{0}}\left(x_{0}\right)}\left|\hat{u}_{v}\right|^{\frac{2(n-1)}{n-2}} d \sigma_{g} \geq \lambda_{0}
$$

up to a subsequence. Now we set

$$
\mu_{v}(t)=\max _{x \in \partial M} \int_{D_{t}(x)}\left|\hat{u}_{v}\right|^{\frac{2(n-1)}{n-2}} d \sigma_{g}
$$

for $t>0$, and, for any $\lambda \in\left(0, \lambda_{0}\right)$, choose sequences $\left\{t_{v}\right\} \subset\left(0, t_{0}\right)$ and $\left\{x_{v}\right\} \subset \partial M$ such that

$$
\lambda=\mu_{v}\left(t_{v}\right)=\int_{D_{t_{v}}\left(x_{v}\right)}\left|\hat{u}_{v}\right|^{\frac{2(n-1)}{n-2}} d \sigma_{g} .
$$

We can also assume that $x_{v}$ converges. Now, we choose $r_{0}>0$ small such that for any $x_{0} \in \partial M$ the Fermi coordinates $\psi_{x_{0}}(z)$ centered at $x_{0}$ are defined for all $z \in B_{2 r_{0}}^{+}(0) \subset \mathbb{R}_{+}^{n}$ and satisfy

$$
\frac{1}{2}\left|z-z^{\prime}\right| \leq d_{g}\left(\psi_{x_{0}}(z), \psi_{x_{0}}\left(z^{\prime}\right)\right) \leq 2\left|z-z^{\prime}\right| \quad \text { for any } z, z^{\prime} \in B_{r_{0}}^{+}(0) .
$$

For each $v$ we consider Fermi coordinates

$$
\psi_{v}=\psi_{x_{v}}: B_{2 r_{0}}^{+}(0) \rightarrow M .
$$

For any $R_{v} \geq 1$ and $y \in B_{R_{v} r_{0}}^{+}(0)$, we set

$$
\tilde{u}_{v}(y)=R_{v}^{-\frac{n-2}{2}} \hat{u}_{v}\left(\psi_{v}\left(R_{v}^{-1} y\right)\right) \quad \text { and } \quad \tilde{g}_{v}(y)=\left(\psi_{v}^{*} g\right)\left(R_{v}^{-1} y\right) .
$$


Let us consider $z \in \mathbb{R}_{+}^{n}$ and $r>0$ such that $|z|+r<R_{\nu} r_{0}$. Then we have

$$
\int_{B_{r}^{+}(z)}\left|d \tilde{u}_{v}\right|_{\tilde{g}_{v}}^{2} d v_{\tilde{g}_{v}}=\int_{\psi_{v}\left(R_{v}^{-1} B_{r}^{+}(z)\right)}\left|d \hat{u}_{v}\right|_{g}^{2} d v_{g}
$$

and, if in addition $z \in \partial \mathbb{R}_{+}^{n}$,

$$
\begin{aligned}
\int_{\partial^{\prime} B_{r}^{+}(z)}\left|\tilde{u}_{\nu}\right|^{\frac{2(n-1)}{n-2}} d \sigma_{\tilde{g}_{\nu}} & =\int_{\psi_{\nu}\left(R_{\nu}^{-1} \partial^{\prime} B_{r}^{+}(z)\right)}\left|\hat{u}_{v}\right|^{\frac{2(n-1)}{n-2}} d \sigma_{g} \\
& \leq \int_{D_{2 R_{\nu}^{-1} r^{\prime}}\left(\psi_{\nu}\left(R_{\nu}^{-1} z\right)\right)}\left|\hat{u}_{v}\right|^{\frac{2(n-1)}{n-2}} d \sigma_{g},
\end{aligned}
$$

where we have used the fact that

$$
\psi_{v}\left(R_{v}^{-1} \partial^{\prime} B_{r}^{+}(z)\right)=\psi_{v}\left(\partial^{\prime} B_{R_{v}^{-1} r}^{+}\left(R_{v}^{-1} z\right)\right) \subset D_{2 R_{\nu}^{-1} r}\left(\psi_{v}\left(R_{v}^{-1} z\right)\right) .
$$

Given $r \in\left(0, r_{0}\right)$ we fix $t_{0} \leq 2 r$. Then, given a $\lambda \in\left(0, \lambda_{0}\right)$ to be fixed later, we set $R_{v}=2 r t_{v}^{-1} \geq 2 r t_{0}^{-1} \geq 1$. It follows from (2-5) and (2-6) that

$$
\int_{\partial^{\prime} B_{r}^{+}(z)}\left|\tilde{u}_{\nu}\right|^{\frac{2(n-1)}{n-2}} d \sigma_{\tilde{g}_{\nu}} \leq \lambda .
$$

Moreover, since $\psi_{\nu}\left(\partial^{\prime} B_{2 R_{v}^{-1} r}^{+}(0)\right)=D_{t_{v}}\left(x_{v}\right)$, we have

$$
\int_{\partial^{\prime} B_{2 r}^{+}(0)}\left|\tilde{u}_{v}\right|^{\frac{2(n-1)}{n-2}} d \sigma_{\tilde{g}_{v}}=\int_{D_{t_{v}}\left(x_{v}\right)}\left|\hat{u}_{\nu}\right|^{\frac{2(n-1)}{n-2}} d \sigma_{g}=\lambda .
$$

Choosing $r_{0}$ smaller if necessary, we can suppose that

$$
\frac{1}{2} \int_{\mathbb{R}_{+}^{n}}|d u|^{2} d y \leq \int_{\mathbb{R}_{+}^{n}}|d u|_{\tilde{g}_{x_{0}, R}}^{2} d v_{\tilde{g}_{x_{0}, R}} \leq 2 \int_{\mathbb{R}_{+}^{n}}|d u|^{2} d y
$$

for any $R \geq 1$ and any $u \in D^{1}\left(\mathbb{R}_{+}^{n}\right) \operatorname{such}$ that $\operatorname{supp}(u) \subset B_{2 r_{0} R}^{+}(0)$. Here, $\tilde{g}_{x_{0}, R}(y)=$ $\left(\psi_{x_{0}}^{*} g\right)\left(R^{-1} y\right)$. We can also assume that

$$
\frac{1}{2} \int_{\partial \mathbb{R}_{+}^{n}}|u| d y \leq \int_{\partial \mathbb{R}_{+}^{n}}|u| d \sigma_{\tilde{g}_{x_{0}, R}} \leq 2 \int_{\partial \mathbb{R}_{+}^{n}}|u| d y
$$

for all $u \in L^{1}\left(\partial \mathbb{R}_{+}^{n}\right)$ such that $\operatorname{supp}(u) \subset \partial^{\prime} B_{2 r_{0} R}^{+}(0)$.

Let $\tilde{\eta}$ be a smooth cutoff function on $\mathbb{R}^{n}$ such that $0 \leq \tilde{\eta} \leq 1, \tilde{\eta}(z)=1$ for $|z| \leq \frac{1}{4}$, and $\tilde{\eta}(z)=0$ for $|z| \geq \frac{3}{4}$. We set $\tilde{\eta}_{v}(y)=\tilde{\eta}\left(r_{0}^{-1} R_{v}^{-1} y\right)$.

It is easy to check that

$$
\left\{\int_{\mathbb{R}_{+}^{n}}\left|d\left(\tilde{\eta}_{v} \tilde{u}_{v}\right)\right|_{\tilde{g}_{v}}^{2} d v_{\tilde{g}_{v}}\right\}
$$

is uniformly bounded. Then the inequality (2-9) implies that $\left\{\tilde{\eta}_{v} \tilde{u}_{v}\right\}$ is uniformly 
bounded in $D^{1}\left(\mathbb{R}_{+}^{n}\right)$ and we can assume that $\tilde{\eta}_{v} \tilde{u}_{v} \rightarrow u$ in $D^{1}\left(\mathbb{R}_{+}^{n}\right)$ for some $u$.

Claim 1. Let us set $r_{1}=r_{0} / 24$. There exists $\lambda_{1}=\lambda_{1}(n)$ such that for any $0<r<r_{1}$ and $0<\lambda<\min \left\{\lambda_{1}, \lambda_{0}\right\}$ we have

$$
\tilde{\eta}_{v} \tilde{u}_{v} \rightarrow u \text { in } H^{1}\left(B_{2 R r}^{+}(0)\right) \text { as } v \rightarrow \infty
$$

for any $R \geq 1$ satisfying $R \leq R_{v}$ for all $v$ large.

Proof. We consider $r \in\left(0, r_{1}\right), \lambda \in\left(0, \lambda_{0}\right)$ and choose $z_{0} \in \partial \mathbb{R}_{+}^{n}$ such that $\left|z_{0}\right|<$ $3(2 R-1) r_{1}$. By Fatou's lemma,

$$
\begin{aligned}
\int_{r}^{2 r} \liminf _{\nu \rightarrow \infty}\left\{\int_{\partial^{+} B_{\rho}^{+}\left(z_{0}\right)}\left(\left|d\left(\tilde{\eta}_{\nu} \tilde{u}_{v}\right)\right|^{2}+\left|\tilde{\eta}_{\nu} \tilde{u}_{v}\right|^{2}\right) d \sigma_{\rho}\right\} d \rho \\
\quad \leq \liminf _{\nu \rightarrow \infty} \int_{B_{2 r}^{+}\left(z_{0}\right)}\left(\left|d\left(\tilde{\eta}_{\nu} \tilde{u}_{\nu}\right)\right|^{2}+\left|\tilde{\eta}_{\nu} \tilde{u}_{v}\right|^{2}\right) d y \leq C,
\end{aligned}
$$

where $d \sigma_{\rho}$ is the volume form on $\partial^{+} B_{\rho}^{+}\left(z_{0}\right)$ induced by the Euclidean metric. Thus there exists $\rho \in[r, 2 r]$ such that, up to a subsequence,

$$
\int_{\partial^{+} B_{\rho}^{+}\left(z_{0}\right)}\left(\left|d\left(\tilde{\eta}_{v} \tilde{u}_{v}\right)\right|^{2}+\left|\tilde{\eta}_{\nu} \tilde{u}_{v}\right|^{2}\right) d \sigma_{\rho} \leq C \quad \text { for all } v .
$$

Hence, $\left\{\left\|\tilde{\eta}_{\nu} \tilde{u}_{v}\right\|_{H^{1}\left(\partial^{+} B_{\rho}^{+}\left(z_{0}\right)\right)}\right\}$ is uniformly bounded, and, since the embedding

$$
H^{1}\left(\partial^{+} B_{\rho}^{+}\left(z_{0}\right)\right) \subset H^{1 / 2}\left(\partial^{+} B_{\rho}^{+}\left(z_{0}\right)\right)
$$

is compact, we can assume that

$$
\tilde{\eta}_{v} \tilde{u}_{v} \rightarrow u \text { in } H^{1 / 2}\left(\partial^{+} B_{\rho}^{+}\left(z_{0}\right)\right) \quad \text { as } v \rightarrow \infty .
$$

We set $\mathscr{A}=B_{3 r}^{+}\left(z_{0}\right)-\overline{B_{\rho}^{+}\left(z_{0}\right)}$, and let $\left\{\phi_{\nu}\right\} \subset D^{1}\left(\mathbb{R}_{+}^{n}\right)$ be such that

$$
\phi_{\nu}= \begin{cases}\tilde{\eta}_{\nu} \tilde{u}_{\nu}-u, & \text { in } B_{\rho+\epsilon}^{+}\left(z_{0}\right), \\ 0, & \text { in } \mathbb{R}_{+}^{n} \backslash B_{3 r-\epsilon}^{+}\left(z_{0}\right),\end{cases}
$$

with $\epsilon>0$ small. Then

$$
\left\|\tilde{\eta}_{\nu} \tilde{u}_{v}-u\right\|_{H^{1 / 2}\left(\partial^{+} B_{\rho}^{+}\left(z_{0}\right)\right)}=\left\|\phi_{\nu}\right\|_{H^{1 / 2}\left(\partial^{+} B_{\rho}^{+}\left(z_{0}\right)\right)} \rightarrow 0 \quad \text { as } v \rightarrow \infty,
$$

and there exists $\left\{\phi_{\nu}^{0}\right\} \subset D^{1}(\mathscr{A})$ such that

$$
\left\|\phi_{v}+\phi_{v}^{0}\right\|_{H^{1}(\mathscr{A})} \leq C\left\|\phi_{v}\right\|_{H^{1 / 2}\left(\partial^{+} \mathscr{A}\right)}=C\left\|\phi_{\nu}\right\|_{H^{1 / 2}\left(\partial^{+} B_{\rho}^{+}\left(z_{0}\right)\right)}
$$

for some $C>0$ independent of $v$. Here, $D^{1}(\mathscr{A})$ is the closure of $C_{0}^{\infty}(\mathscr{A})$ in $H^{1}(\mathscr{A})$, and we have set $\partial^{+} \mathscr{A}=\partial \mathscr{A} \cap\left(\mathbb{R}_{+}^{n} \backslash \partial \mathbb{R}_{+}^{n}\right)$ and $\partial^{\prime} \mathscr{A}=\partial \mathscr{A} \cap \partial \mathbb{R}_{+}^{n}$.

The sequence of functions $\left\{\zeta_{v}\right\}=\left\{\phi_{v}+\phi_{v}^{0}\right\} \subset D^{1}\left(\mathbb{R}_{+}^{n}\right)$ satisfies 


$$
\zeta_{v}= \begin{cases}\tilde{\eta}_{v} \tilde{u}_{v}-u & \text { in } \overline{B_{\rho}^{+}\left(z_{0}\right)} \\ \phi_{v}+\phi_{v}^{0} & \text { in } B_{3 r}^{+}\left(z_{0}\right) \backslash \overline{B_{\rho}^{+}\left(z_{0}\right)} \\ 0 & \text { in } \mathbb{R}_{+}^{n} \backslash B_{3 r}^{+}\left(z_{0}\right)\end{cases}
$$

In particular, $\zeta_{v} \rightarrow 0$ in $H^{1}(\mathscr{A})$. We set

$$
\tilde{\zeta}_{\nu}(x)=R_{\nu}^{\frac{n-2}{2}} \zeta_{v}\left(R_{v} \psi_{\nu}^{-1}(x)\right) \text { if } x \in \psi_{\nu}\left(B_{6 r_{1}}^{+}(0)\right),
$$

and $\tilde{\zeta}_{v}(x)=0$ otherwise. Since we are assuming $\left|z_{0}\right|<3(2 R-1) r_{1} \leq 3\left(2 R_{v}-1\right) r_{1}$ for all $v$ large, $B_{3 r}^{+}\left(z_{0}\right) \subset B_{6 r_{1} R_{v}}^{+}(0)$. Hence,

$$
\tilde{\zeta}_{v}(x)= \begin{cases}R_{v}^{\frac{n-2}{2}}\left(\tilde{\eta}_{v} \tilde{u}_{v}-u\right)\left(R_{v} \psi_{v}^{-1}(x)\right) & \text { if } x \in \psi_{v}\left(R_{v}^{-1} \overline{B_{\rho}^{+}\left(z_{0}\right)}\right), \\ R_{v}^{\frac{n-2}{2}}\left(\phi_{v}+\phi_{v}^{0}\right)\left(R_{v} \psi_{v}^{-1}(x)\right) & \text { if } \left.x \in \psi_{v}\left(R_{v}^{-1} \overline{\left(B_{3 r}^{+}\left(z_{0}\right)\right.} \backslash B_{\rho}^{+}\left(z_{0}\right)\right)\right),\end{cases}
$$

and $\tilde{\zeta}_{\nu}(x)=0$ otherwise, and

$(2-11) d I_{g}\left(\hat{u}_{v}\right) \cdot \tilde{\zeta}_{v}$

$$
\begin{aligned}
& =d I_{g}\left(\hat{\eta}_{v} \hat{u}_{v}\right) \cdot \tilde{\zeta}_{v} \\
& =\int_{B_{3 r}^{+}\left(z_{0}\right)}\left\langle d\left(\tilde{\eta}_{\nu} \tilde{u}_{v}\right), d \zeta_{\nu}\right\rangle_{\tilde{g}_{v}} d v_{\tilde{g}_{v}}-\int_{\partial^{\prime} B_{3 r}^{+}\left(z_{0}\right)}\left|\tilde{\eta}_{v} \tilde{u}_{v}\right|^{\frac{2}{n-2}}\left(\tilde{\eta}_{\nu} \tilde{u}_{v}\right) \zeta_{v} d \sigma_{\tilde{g}_{v}},
\end{aligned}
$$

where $\hat{\eta}_{v}(x)=\tilde{\eta}\left(r_{0}^{-1} \psi_{v}^{-1}(x)\right)$.

Since there exists $C>0$ such that $\left\|\tilde{\zeta}_{\nu}\right\|_{H^{1}(M)} \leq C\left\|\zeta_{\nu}\right\|_{D^{1}\left(\mathbb{R}_{+}^{n}\right)}$, the sequence $\left\{\tilde{\zeta}_{v}\right\}$ is uniformly bounded in $H^{1}(M)$. Hence,

$$
d I_{g}\left(\hat{u}_{v}\right) \cdot \tilde{\zeta}_{v} \rightarrow 0 \quad \text { as } v \rightarrow \infty
$$

Noting that $\zeta_{v} \rightarrow 0$ in $H^{1}(\mathscr{A})$ and $\zeta_{v} \rightarrow 0$ in $D^{1}\left(\mathbb{R}_{+}^{n}\right)$, we obtain

$$
\begin{aligned}
\int_{B_{3 r}^{+}\left(z_{0}\right)}\left\langle d\left(\tilde{\eta}_{\nu} \tilde{u}_{v}\right), d \zeta_{\nu}\right\rangle_{\tilde{g}_{\nu}} d v_{\tilde{g}_{\nu}} & =\int_{B_{\rho}^{+}\left(z_{0}\right)}\left\langle d\left(\zeta_{\nu}+u\right), d \zeta_{\nu}\right\rangle_{\tilde{g}_{\nu}} d v_{\tilde{g}_{\nu}}+o(1) \\
& =\int_{\mathbb{R}_{+}^{n}}\left|d \zeta_{\nu}\right|_{\tilde{g}_{\nu}}^{2} d v_{\tilde{g}_{\nu}}+o(1)
\end{aligned}
$$

Similarly,

$$
\int_{\partial^{\prime} B_{3 r}^{+}\left(z_{0}\right)}\left|\tilde{\eta}_{\nu} \tilde{u}_{v}\right|^{\frac{2}{n-2}}\left(\tilde{\eta}_{\nu} \tilde{u}_{v}\right) \zeta_{\nu} d \sigma_{\tilde{g}_{v}}=\int_{\partial \mathbb{R}_{+}^{n}}\left|\zeta_{v}\right|^{\frac{2(n-1)}{n-2}} d \sigma_{\tilde{g}_{\nu}}+o(1) .
$$

Using (2-11), (2-12), (2-13) and (2-14) we conclude that

$$
\int_{\mathbb{R}_{+}^{n}}\left|d \zeta_{v}\right|_{\tilde{g}_{v}}^{2} d v_{\tilde{g}_{v}}=\int_{\partial \mathbb{R}_{+}^{n}}\left|\zeta_{v}\right|^{\frac{2(n-1)}{n-2}} d \sigma_{\tilde{g}_{v}}+o(1) .
$$


Using again the facts that $\zeta_{v} \rightarrow 0$ in $H^{1}(\mathscr{A})$ and $\zeta_{v} \rightarrow 0$ in $D^{1}\left(\mathbb{R}_{+}^{n}\right)$, we can apply the inequality

$$
\begin{aligned}
&|| \tilde{\eta}_{\nu} \tilde{u}_{v}-\left.u\right|^{\frac{2(n-1)}{n-2}}-\left|\tilde{\eta}_{\nu} \tilde{u}_{v}\right|^{\frac{2(n-1)}{n-2}}+|u|^{\frac{2(n-1)}{n-2}} \mid \\
& \leq C|u|^{\frac{n}{n-2}}\left|\tilde{\eta}_{\nu} \tilde{u}_{v}-u\right|+C\left|\tilde{\eta}_{\nu} \tilde{u}_{\nu}-u\right|^{\frac{n}{n-2}}|u|
\end{aligned}
$$

to see that

$$
\int_{\partial \mathbb{R}_{+}^{n}}\left|\zeta_{\nu}\right|^{\frac{2(n-1)}{n-2}} d \sigma_{\tilde{g}_{\nu}}=\int_{\partial^{\prime} B_{\rho}^{+}\left(z_{0}\right)}\left|\tilde{\eta}_{\nu} \tilde{u}_{\nu}\right|^{\frac{2(n-1)}{n-2}} d \sigma_{\tilde{g}_{\nu}}-\int_{\partial^{\prime} B_{\rho}^{+}\left(z_{0}\right)}|u|^{\frac{2(n-1)}{n-2}} d \sigma_{\tilde{g}_{\nu}}+o(1) .
$$

This implies

$$
\begin{aligned}
\int_{\partial \mathbb{R}_{+}^{n}}\left|\zeta_{v}\right|^{\frac{2(n-1)}{n-2}} d \sigma_{\tilde{g}_{v}} & \leq \int_{\partial^{\prime} B_{\rho}^{+}\left(z_{0}\right)}\left|\tilde{\eta}_{v} \tilde{u}_{v}\right|^{\frac{2(n-1)}{n-2}} d \sigma_{\tilde{g}_{v}}+o(1) \\
& =\int_{\partial^{\prime} B_{\rho}^{+}\left(z_{0}\right)}\left|\tilde{u}_{v}\right|^{\frac{2(n-1)}{n-2}} d \sigma_{\tilde{g}_{v}}+o(1)
\end{aligned}
$$

where we have used the fact that $\tilde{\eta}_{\nu}(z)=1$ for all $z \in B_{\rho}^{+}\left(z_{0}\right)$.

If $N=N(n) \in \mathbb{N}$ is such that $\partial^{\prime} B_{2}^{+}(0)$ is covered by $N$ discs in $\partial \mathbb{R}_{+}^{n}$ of radius 1 with center in $\partial^{\prime} B_{2}^{+}(0)$, then we can choose points $z_{i} \in \partial^{\prime} B_{2 r}^{+}\left(z_{0}\right), i=1, \ldots, N$, satisfying

$$
\partial^{\prime} B_{\rho}^{+}\left(z_{0}\right) \subset \partial^{\prime} B_{2 r}^{+}\left(z_{0}\right) \subset \bigcup_{i=1}^{N} \partial^{\prime} B_{r}^{+}\left(z_{i}\right) .
$$

Hence, using (2-7), (2-15) and (2-16), we see that

$$
\int_{\mathbb{R}_{+}^{n}}\left|d \zeta_{v}\right|_{\tilde{g}_{v}}^{2} d v_{\tilde{g}_{v}}+o(1)=\int_{\partial \mathbb{R}_{+}^{n}}\left|\zeta_{v}\right|^{\frac{2(n-1)}{n-2}} d \sigma_{\tilde{g}_{v}} \leq N \lambda+o(1)
$$

It follows from (2-9), (2-10) and the Sobolev inequality (1-6) that

$$
\begin{aligned}
\left(\int_{\partial \mathbb{R}_{+}^{n}}\left|\zeta_{\nu}\right|^{\frac{2(n-1)}{n-2}} d \sigma_{\tilde{g}_{\nu}}\right)^{\frac{n-2}{n-1}} & \leq 2^{\frac{n-2}{n-1}}\left(\int_{\partial \mathbb{R}_{+}^{n}}\left|\zeta_{\nu}\right|^{\frac{2(n-1)}{n-2}} d x\right)^{\frac{n-2}{n-1}} \\
& \leq 2^{\frac{n-2}{n-1}} K_{n}^{2} \int_{\mathbb{R}_{+}^{n}}\left|d \zeta_{\nu}\right|^{2} d x \\
& \leq 2^{1+\frac{n-2}{n-1}} K_{n}^{2} \int_{\mathbb{R}_{+}^{n}}\left|d \zeta_{\nu}\right|_{\tilde{g}_{v}}^{2} d v_{\tilde{g}_{v}}
\end{aligned}
$$

Then using (2-15) and (2-17) we obtain 


$$
\begin{aligned}
\int_{\mathbb{R}_{+}^{n}}\left|d \zeta_{\nu}\right|_{\tilde{g}_{\nu}}^{2} d v_{\tilde{g}_{\nu}} & =\int_{\partial \mathbb{R}_{+}^{n}}\left|\zeta_{\nu}\right|^{\frac{2(n-1)}{n-2}} d \sigma_{\tilde{g}_{\nu}}+o(1) \\
& \leq\left(2^{1+\frac{n-2}{n-1}} K_{n}^{2}\right)^{\frac{n-1}{n-2}}\left(\int_{\mathbb{R}_{+}^{n}}\left|d \zeta_{\nu}\right|_{\tilde{g}_{\nu}}^{2} d v_{\tilde{g}_{\nu}}\right)^{\frac{n-1}{n-2}}+o(1) \\
& \leq 2^{1+\frac{n-1}{n-2}} K_{n}^{\frac{2(n-1)}{n-2}}(N \lambda+o(1))^{\frac{1}{n-2}} \int_{\mathbb{R}_{+}^{n}}\left|d \zeta_{\nu}\right|_{\tilde{g}_{\nu}}^{2} d v_{\tilde{g}_{\nu}}+o(1)
\end{aligned}
$$

Now we set $\lambda_{1}=\frac{K_{n}^{-2(n-1)}}{2^{2 n-3} N}$ and assume that $\lambda<\lambda_{1}$. Then

$$
2^{1+\frac{n-1}{n-2}}(N \lambda)^{\frac{1}{n-2}} K_{n}^{\frac{2(n-1)}{n-2}}<1,
$$

and we conclude that

$$
\lim _{\nu \rightarrow \infty} \int_{\mathbb{R}_{+}^{n}}\left|d \zeta_{\nu}\right|_{\tilde{g}_{\nu}}^{2} d v_{\tilde{g}_{v}}=0
$$

Hence, $\zeta_{v} \rightarrow 0$ in $D^{1}\left(\mathbb{R}_{+}^{n}\right)$. Since $r \leq \rho$, we have

$$
\tilde{\eta}_{v} \tilde{u}_{v} \rightarrow u \quad \text { in } H^{1}\left(B_{r}^{+}\left(z_{0}\right)\right)
$$

Now let us choose any $z_{0}=\left(\left(z_{0}\right)^{1}, \ldots,\left(z_{0}\right)^{n}\right) \in \mathbb{R}_{+}^{n}$ satisfying $\left(z_{0}\right)^{n}>r / 2$ and $\left|z_{0}\right|<3(2 R-1) r_{1}$. Using this choice of $z_{0}$ and $r^{\prime}=r / 6$ replacing $r$, the process above can be performed with some obvious modifications. In this case, we have $\partial^{\prime} B_{3 r^{\prime}}^{+}\left(z_{0}\right)=\varnothing$ and the boundary integrals vanish. Hence, the equality (2-15) already implies that $\tilde{\eta}_{v} \tilde{u}_{v} \rightarrow u$ in $H^{1}\left(B_{r^{\prime}}^{+}\left(z_{0}\right)\right)$.

If $N_{1}=N_{1}(R, n) \in \mathbb{N}$ and $N_{2}=N_{2}(R, n) \in \mathbb{N}$ are such that the half-ball $B_{2 R}^{+}(0)$ is covered by $N_{1}$ half-balls of radius 1 with centers in $\partial^{\prime} B_{2 R}^{+}(0)$, plus $N_{2}$ balls of radius $1 / 6$ with centers in $\left\{z=\left(z^{1}, \ldots, z^{n}\right) \in B_{2 R}^{+}(0): z^{n}>1 / 2\right\}$, then the half-ball $B_{2 R r}^{+}(0)$ is covered by $N_{1}$ half-balls of radius $r$ with centers in $\partial^{\prime} B_{2 R r}^{+}(0)$, plus $N_{2}$ balls of radius $r / 6$ with center in $\left\{z=\left(z^{1}, \ldots, z^{n}\right) \in B_{2 R r}^{+}(0): z^{n}>r / 2\right\}$.

Hence, $\tilde{\eta}_{v} \tilde{u}_{v} \rightarrow u$ in $H^{1}\left(B_{2 R r}^{+}(0)\right)$, finishing the proof of Claim 1.

Using (2-8), (2-10) and Claim 1 with $R=1$, we see that

$$
\begin{aligned}
\lambda & =\int_{\partial^{\prime} B_{r}^{+}(0)}\left|\tilde{u}_{\nu}\right|^{\frac{2(n-1)}{n-2}} d \sigma_{\tilde{g}_{\nu}} \\
& =\int_{\partial^{\prime} B_{r}^{+}(0)}\left|\tilde{\eta}_{\nu} \tilde{u}_{\nu}\right|^{\frac{2(n-1)}{n-2}} d \sigma_{\tilde{g}_{\nu}} \\
& \leq 2 \int_{\partial^{\prime} B_{r}^{+}(0)}|u|^{\frac{2(n-1)}{n-2}} d x+o(1) .
\end{aligned}
$$

It follows that $u \neq \equiv 0$, due to (1-6). 
Claim 2. We have $\lim _{v \rightarrow \infty} R_{v}=\infty$. In particular, Claim 1 can be stated for any $R \geq 1$.

Proof. Suppose for a contradiction that, up to a subsequence, $R_{v} \rightarrow R^{\prime}$ as $v \rightarrow \infty$, for some $1 \leq R^{\prime}<\infty$. Then, since $\hat{u}_{v} \rightarrow 0$ in $H^{1}(M)$, we have $\tilde{u}_{v} \rightarrow 0$ in $H^{1}\left(B_{2 r}^{+}(0)\right)$. This contradicts the fact that

$$
\tilde{u}_{v} \tilde{\eta}_{v} \rightarrow u \not \equiv 0 \text { in } H^{1}\left(B_{2 r}^{+}(0)\right)
$$

which is obtained by applying Claim 1 with $R=1$. This proves Claim 2 .

That $u$ is a (weak) solution of (2-4) follows easily from the fact that $\left\{\hat{u}_{v}\right\}$ is a Palais-Smale sequence for $I_{g}$ and $\tilde{\eta}_{v} \tilde{u}_{v} \rightarrow u$ in $D^{1}\left(\mathbb{R}_{+}^{n}\right)$.

Now, we set

$$
V_{v}(x)=\eta_{v}(x) R_{v}^{\frac{n-2}{2}} u\left(R_{\nu} \psi_{x_{v}}^{-1}(x)\right)
$$

for $x \in \psi_{x_{v}}\left(B_{2 r_{0}}^{+}(0)\right)$, and 0 otherwise. The proof of the following claim is totally analogous to step 3 on p. 37 of [Druet et al. 2004] with some obvious modifications.

Claim 3. We have $\hat{u}_{v}-V_{v} \rightarrow 0$, as $v \rightarrow \infty$, in $H^{1}(M)$. Moreover, as $v \rightarrow \infty$,

$$
d I_{g}\left(V_{v}\right) \rightarrow 0 \quad \text { and } \quad d I_{g}\left(\hat{u}_{v}-V_{v}\right) \rightarrow 0
$$

strongly in $H^{1}(M)^{\prime}$, and

$$
I_{g}\left(\hat{u}_{v}\right)-I_{g}\left(\hat{u}_{v}-V_{v}\right) \rightarrow E(u) .
$$

We finally observe that if $r_{0}^{\prime}>0$ is also sufficiently small then $\left|\left(\eta_{v}-\eta_{v}^{\prime}\right) V_{v}\right| \rightarrow 0$ as $v \rightarrow \infty$, where $\eta_{v}^{\prime}$ is a smooth cutoff function such that $\eta_{v}^{\prime} \equiv 1$ in $\psi_{x_{v}}\left(B_{r_{0}^{\prime}}^{+}(0)\right)$ and $\eta_{v}^{\prime} \equiv 0$ in $M \backslash \psi_{x_{v}}\left(B_{2 r_{0}^{\prime}}^{+}(0)\right)$. Hence, the statement of Lemma 2.4 holds for any $r_{0}>0$ sufficiently small, finishing the proof.

Proof of Theorem 1.3. According to Lemma 2.1, the Palais-Smale sequence $\left\{u_{v}\right\}$ for $I_{g}^{v}$ is uniformly bounded in $H^{1}(M)$. Hence, we can assume that $u_{v} \rightarrow u^{0}$ in $H^{1}(M)$, and $u_{v} \rightarrow u^{0}$ a.e. in $M$, for some $0 \leq u^{0} \in H^{1}(M)$. By Lemma 2.2, $u^{0}$ is a solution to the equations (1-3). Moreover, $\hat{u}_{v}=u_{v}-u^{0}$ is Palais-Smale for $I_{g}$ and satisfies

$$
I_{g}\left(\hat{u}_{v}\right)=I_{g}^{v}\left(u_{v}\right)-I_{g}^{\infty}\left(u^{0}\right)+o(1) .
$$

If $\hat{u}_{v} \rightarrow 0$ in $H^{1}(M)$, then the theorem is proved. If $\hat{u}_{v} \rightarrow 0$ in $H^{1}(M)$ but not strongly, then we apply Lemma 2.4 to obtain a new Palais-Smale sequence $\left\{\hat{u}_{v}^{1}\right\}$ satisfying

$$
I_{g}\left(\hat{u}_{v}^{1}\right) \leq I_{g}\left(\hat{u}_{v}\right)-\beta^{*}+o(1)=I_{g}^{v}\left(u_{v}\right)-I_{g}^{\infty}\left(u^{0}\right)-\beta^{*}+o(1),
$$

where $\beta^{*}=\frac{K_{n}^{-2(n-1)}}{2(n-1)}$. The term $\beta^{*}$ appears in this inequality because $E(u) \geq \beta^{*}$ 
for any nontrivial solution $u \in D^{1}\left(\mathbb{R}_{+}^{n}\right)$ to the equations (1-1). This can be seen using the Sobolev inequality (1-6).

Now we again have either $\hat{u}_{v}^{1} \rightarrow 0$ in $H^{1}(M)$, in which case the theorem is proved, or we apply Lemma 2.4 to obtain a new Palais-Smale sequence $\left\{\hat{u}_{v}^{2}\right\}$. The process follows by induction and stops, by virtue of Lemma 2.3 , once we obtain a Palais-Smale sequence $\left\{\hat{u}_{v}^{m}\right\}$ with $I_{g}\left(\hat{u}_{v}^{m}\right)$ converging to some $\beta<\beta^{*}$.

We are now left with the proof of (1-7) and the fact that the $U^{j}$ obtained by the process above are of the form (1-5). To that end, we can follow the proof of Lemma 3.3 in [Druet et al. 2004], with some simple changes, to obtain the relation (1-7) and to prove that the $U^{j}$ are nonnegative. For the reader's convenience this is outlined below.

Claim. The functions $u^{0}$ and $U^{j}$ obtained above are nonnegative. Moreover, the identity (1-7) holds.

Proof. That $u^{0}$ is nonnegative is straightforward. To prove that the $U^{j}$ are also nonnegative, set $\hat{u}_{v}=u_{v}-u^{0}$ and $\mu_{v}^{j}=1 / R_{v}^{j}$.

Given integers $N \in[1, m]$ and $s \in[0, N-1]$, we will prove that there exist an integer $p$ and sequences $\left\{\tilde{x}_{v}^{k}\right\}_{v \in \mathbb{N}} \subset \partial M$ and $\left\{\lambda_{v}^{k}>0\right\}_{v \in \mathbb{N}}$ for each $k=1, \ldots, p$, such that $d_{g}\left(x_{v}^{N}, \tilde{x}_{v}^{k}\right) / \mu_{v}^{N}$ is bounded and $\lim _{v \rightarrow \infty} \lambda_{v}^{k} / \mu_{v}^{N}=0$, and such that

$$
\int_{\Omega_{v}^{N}(R) \backslash \bigcup_{k=1}^{p} \widetilde{\Omega}_{v}^{k}\left(R^{\prime}\right)}\left|\hat{u}_{v}-\sum_{j=1}^{s} u_{v}^{j}-u_{v}^{N}\right|^{\frac{2 n}{n-2}} d v_{g}=o(1)+\epsilon\left(R^{\prime}\right)
$$

for any $R, R^{\prime}>0$. Here, $\Omega_{v}^{N}(R)=\psi_{x_{v}^{N}}\left(B_{R \mu_{v}^{N}}^{+}(0)\right), \widetilde{\Omega}_{v}^{k}\left(R^{\prime}\right)=\psi_{\tilde{x}_{v}^{k}}\left(B_{R^{\prime} \lambda_{v}^{k}}^{+}(0)\right)$ and $\epsilon\left(R^{\prime}\right) \rightarrow 0$ as $R^{\prime} \rightarrow \infty$.

We prove (2-20) by reverse induction on $s$. It follows from Claim 2 in the proof of Lemma 2.4 that

$$
\int_{\Omega_{v}^{N}(R)}\left|\hat{u}_{v}-\sum_{j=1}^{N-1} u_{v}^{j}-u_{v}^{N}\right|^{\frac{2 n}{n-2}} d v_{g}=o(1),
$$

so that (2-20) holds for $s=N-1$.

Assuming (2-20) holds for some $s \in[1, N-1]$, let us prove it does for $s-1$.

We first consider the case when $d_{g}\left(x_{v}^{s}, x_{v}^{N}\right)$ does not converge to zero as $v \rightarrow \infty$. In this case, we can assume $\Omega_{v}^{N}(R) \cap \Omega_{v}^{s}(\widetilde{R})=\varnothing$ for any $\widetilde{R}>0$. Then after rescaling we have

$$
\int_{\Omega_{\nu}^{N}(R) \backslash \bigcup_{k=1}^{p} \widetilde{\Omega}_{\nu}^{k}\left(R^{\prime}\right)}\left|u_{v}^{s}\right|^{\frac{2 n}{n-2}} d v_{g} \leq C \int_{\mathbb{R}_{+}^{n} \backslash B_{\widetilde{R}}^{+}(0)}\left|U^{s}\right|^{\frac{2 n}{n-2}} d y .
$$

Since $\widetilde{R}>0$ is arbitrary and $U^{s} \in L^{\frac{2 n}{n-2}}\left(\mathbb{R}_{+}^{n}\right)$, the left side of (2-21) converges to zero as $v \rightarrow \infty$. Hence, (2-20) still holds replacing $s$ by $s-1$. 
Now consider the case when $d_{g}\left(x_{v}^{s}, x_{v}^{N}\right) \rightarrow 0$ as $v \rightarrow \infty$. According to Claim 2 in the proof of Lemma 2.4, given $\widetilde{R}>0$, we have

$$
\int_{\Omega_{\nu}^{s}(\widetilde{R})}\left|\hat{u}_{\nu}-\sum_{j=1}^{s} u_{\nu}^{j}\right|^{\frac{2 n}{n-2}} d v_{g}=o(1) .
$$

Using the induction hypothesis (2-20), we then conclude that

$$
\int_{\left(\Omega_{\nu}^{N}(R) \backslash \bigcup_{k=1}^{p} \tilde{\Omega}_{\nu}^{k}\left(R^{\prime}\right)\right) \cap \Omega_{\nu}^{S}(\widetilde{R})}\left|u_{v}^{N}\right|^{\frac{2 n}{n-2}} d v_{g}=o(1)+\epsilon\left(R^{\prime}\right) .
$$

First assume that $d_{g}\left(x_{v}^{s}, x_{v}^{N}\right) / \mu_{v}^{N} \rightarrow \infty$. Rescaling by $\mu_{v}^{N}$ and using coordinates centered at $x_{v}^{N}$, it's not difficult to see that $d_{g}\left(x_{v}^{s}, x_{v}^{N}\right) / \mu_{v}^{s} \rightarrow \infty$. Hence we can assume that $\Omega_{v}^{N}(R) \cap \Omega_{v}^{s}(\widetilde{R})=\varnothing$ for any $\widetilde{R}>0$, and we proceed as in the case when $d_{g}\left(x_{v}^{s}, x_{v}^{N}\right)$ does not converge to 0 to conclude that (2-20) holds for $s-1$.

If $d_{g}\left(x_{v}^{s}, x_{v}^{N}\right) / \mu_{v}^{N}$ does not go to infinity, we can assume that it converges. One can then check that $\mu_{v}^{s} / \mu_{v}^{N} \rightarrow 0$. We set $\tilde{x}_{v}^{p+1}=x_{v}^{s}$ and $\lambda_{v}^{p+1}=\mu_{v}^{s}$, so that $\lambda_{v}^{p+1} / \mu_{v}^{N} \rightarrow 0$ as $v \rightarrow \infty$. Observing that

$$
\int_{\Omega_{\nu}^{N}(R) \backslash \bigcup_{k=1}^{p+1} \tilde{\Omega}_{\nu}^{k}\left(R^{\prime}\right)}\left|u_{v}^{s}\right|^{\frac{2 n}{n-2}} d v_{g} \leq \int_{M \backslash \Omega_{\nu}^{s}\left(R^{\prime}\right)}\left|u_{v}^{s}\right|^{\frac{2 n}{n-2}} d v_{g} \leq \epsilon\left(R^{\prime}\right),
$$

it follows that (2-20) holds when we replace $p$ by $p+1$ and $s$ by $s-1$.

This proves (2-20). The above also proves (1-7).

We fix an integer $N \in[1, m]$ and $s=0$. Let $\tilde{y}_{v}^{k} \in \partial \mathbb{R}_{+}^{n}$ be such that $\tilde{x}_{v}^{k}=$ $\psi_{x_{v}^{N}}^{N}\left(\mu_{v}^{N} \tilde{y}_{v}^{k}\right)$, for $k=1, \ldots, p$. For each $k$, the sequence $\left\{\tilde{y}_{v}^{k}\right\}_{v \in \mathbb{N}}$ is bounded, so there exists $\tilde{y}^{k} \in \partial \mathbb{R}_{+}^{n}$ such that $\lim _{v \rightarrow \infty} \tilde{y}_{v}^{k}=\tilde{y}^{k}$, possibly after taking a subsequence. We set $\tilde{X}=\bigcup_{k=1}^{p} \tilde{y}^{k}$ and

$$
\tilde{u}_{v}^{N}(y)=\left(\mu_{v}^{N}\right)^{\frac{n-2}{2}} \hat{u}_{v}^{N}\left(\psi_{x_{v}^{N}}\left(\mu_{v}^{N} y\right)\right) .
$$

It follows from (2-20) that

$$
\tilde{u}_{v}^{N} \rightarrow U^{N} \quad \text { in } L_{\text {loc }}^{\frac{2 n}{n-2}}\left(B_{R}^{+}(0) \backslash \tilde{X}\right) \quad \text { as } v \rightarrow \infty .
$$

Therefore we can assume that $\tilde{u}_{v} \rightarrow U^{N}$ a.e. in $\mathbb{R}_{+}^{n}$ as $v \rightarrow \infty$.

If we set

$$
\tilde{u}_{v}^{0, N}(y)=\left(\mu_{v}^{N}\right)^{\frac{n-2}{2}} u^{0}\left(\psi_{x_{v}^{N}}\left(\mu_{v}^{N} y\right)\right),
$$

it's easy to prove that

$$
\tilde{u}_{v}^{0, N} \rightarrow 0 \quad \text { in } L_{\text {loc }}^{\frac{2 n}{n-2}}\left(B_{R}^{+}(0)\right) \quad \text { as } v \rightarrow \infty .
$$


Hence, $\tilde{u}_{v}^{0, N} \rightarrow 0$ a.e. in $\mathbb{R}_{+}^{n}$ as $v \rightarrow \infty$. Setting

$$
v_{v}^{N}(y)=\left(\mu_{v}^{N}\right)^{\frac{n-2}{2}} u_{v}^{N}\left(\psi_{x_{v}^{N}}\left(\mu_{v}^{N} y\right)\right),
$$

we see that $v_{v}^{N} \rightarrow U^{N}$ a.e. in $\mathbb{R}_{+}^{n}$ as $v \rightarrow \infty$. In particular, $U^{N}$ is nonnegative. This proves the claim.

Remark 2.5. For the regularity of the $U^{j}$ we can use [Cherrier 1984, théorème 1]. Although that theorem was established for compact manifolds, we can use the conformal equivalence between $\mathbb{R}_{+}^{n}$ and $B^{n} \backslash\{$ point $\}$ and a removable singularities theorem (see Lemma 2.7 on p. 1821 of [Almaraz 2011]) to apply it in $B^{n}$.

Thus we are able to use the result in [Li and Zhu 1995] to conclude that the $U^{j}$ are of the form (1-5), so we can write $U^{j}=U_{\epsilon_{j}, a_{j}}$.

This finishes the proof of Theorem 1.3.

\section{Acknowledgment}

I am grateful to the hospitality of the Department of Mathematics of the Imperial College London, where part of this work was written during the spring of 2012.

\section{References}

[Almaraz 2011] S. Almaraz, "Blow-up phenomena for scalar-flat metrics on manifolds with boundary”, J. Differential Equations 251:7 (2011), 1813-1840. MR 2823676 Zbl 1223.53031

[Almaraz 2012] S. Almaraz, "Convergence of scalar-flat metrics on manifolds with boundary under the Yamabe flow", preprint, 2012. arXiv 1206.1184

[Bahri and Coron 1988] A. Bahri and J.-M. Coron, "On a nonlinear elliptic equation involving the critical Sobolev exponent: the effect of the topology of the domain", Comm. Pure Appl. Math. 41:3 (1988), 253-294. MR 89c:35053 Zbl 0649.35033

[Beckner 1993] W. Beckner, "Sharp Sobolev inequalities on the sphere and the Moser-Trudinger inequality”, Ann. of Math. (2) 138:1 (1993), 213-242. MR 94m:58232 Zbl 0826.58042

[Brendle 2002] S. Brendle, "A generalization of the Yamabe flow for manifolds with boundary", Asian J. Math. 6:4 (2002), 625-644. MR 2003m:53052 Zbl 1039.53035

[Brezis and Coron 1985] H. Brezis and J.-M. Coron, "Convergence of solutions of $H$-systems or how to blow bubbles", Arch. Rational Mech. Anal. 89 (1985), 21-56. MR 86g:53007 Zbl 0584.49024

[Cao et al. 2001] D. Cao, E. S. Noussair, and S. Yan, "Existence and nonexistence of interior-peaked solution for a nonlinear Neumann problem", Pacific J. Math. 200:1 (2001), 19-41. MR 2002i:35065 Zbl 1140.35440

[Chabrowski and Girão 2002] J. Chabrowski and P. M. Girão, "Symmetric solutions of the Neumann problem involving a critical Sobolev exponent", Topol. Methods Nonlinear Anal. 19:1 (2002), 1-27. MR 2003e:35081 Zbl 1029.35088

[Cherrier 1984] P. Cherrier, "Problèmes de Neumann non linéaires sur les variétés Riemanniennes", J. Funct. Anal. 57:2 (1984), 154-206. MR 86c:58154 Zbl 0552.58032 
[Chipot et al. 1996] M. Chipot, I. Shafrir, and M. Fila, "On the solutions to some elliptic equations with nonlinear Neumann boundary conditions", Adv. Differential Equations 1:1 (1996), 91-110. MR 96h:35059 Zbl 0839.35042

[Druet et al. 2004] O. Druet, E. Hebey, and F. Robert, Blow-up theory for elliptic PDEs in Riemannian geometry, Mathematical Notes 45, Princeton University Press, 2004. MR 2005g:53058 Zbl 1059.58017

[Escobar 1988] J. F. Escobar, "Sharp constant in a Sobolev trace inequality", Indiana Univ. Math. J. 37:3 (1988), 687-698. MR 90a:46071 Zbl 0666.35014

[Escobar 1990] J. F. Escobar, "Uniqueness theorems on conformal deformation of metrics, Sobolev inequalities, and an eigenvalue estimate", Comm. Pure Appl. Math. 43:7 (1990), 857-883. MR 92f: 58038 Zbl 0713.53024

[Escobar 1992] J. F. Escobar, "Conformal deformation of a Riemannian metric to a scalar flat metric with constant mean curvature on the boundary", Ann. of Math. (2) 136:1 (1992), 1-50. MR 93e:53046 Zbl 0766.53033

[Li and Zhu 1995] Y. Li and M. Zhu, "Uniqueness theorems through the method of moving spheres", Duke Math. J. 80:2 (1995), 383-417. MR 96k:35061 Zbl 0846.35050

[Li and Zhu 1997] Y. Li and M. Zhu, "Sharp Sobolev trace inequalities on Riemannian manifolds with boundaries”, Comm. Pure Appl. Math. 50:5 (1997), 449-487. MR 98c:46065 Zbl 0869.58054

[Pierotti and Terracini 1995] D. Pierotti and S. Terracini, "On a Neumann problem with critical exponent and critical nonlinearity on the boundary", Comm. Partial Differential Equations 20:7-8 (1995), 1155-1187. MR 96f:35060 Zbl 0829.35038

[Struwe 1984] M. Struwe, "A global compactness result for elliptic boundary value problems involving limiting nonlinearities", Math. Z. 187:4 (1984), 511-517. MR 86k:35046 Zbl 0535.35025

Received November 9, 2012. Revised August 2, 2013.

\author{
SÉrgio Almaraz \\ INSTITUTO DE MATEMÁTICA \\ UNIVERSIDAdE FEDERAL FLUMINENSE \\ Rua Mário SANTOS Braga S/N VALONGUinho \\ 24020-140 \\ NiteróI, RIO DE JANEIRO \\ BRAZIL \\ almaraz@vm.uff.br
}




\title{
PACIFIC JOURNAL OF MATHEMATICS
}

\author{
msp.org/pjm
}

Founded in 1951 by E. F. Beckenbach (1906-1982) and F. Wolf (1904-1989)

\section{EDITORS}

Don Blasius (Managing Editor)

Department of Mathematics

University of California

Los Angeles, CA 90095-1555

blasius@math.ucla.edu

\author{
Paul Balmer \\ Department of Mathematics \\ University of California \\ Los Angeles, CA 90095-1555 \\ balmer@math.ucla.edu \\ Robert Finn \\ Department of Mathematics \\ Stanford University \\ Stanford, CA 94305-2125 \\ finn@math.stanford.edu \\ Sorin Popa \\ Department of Mathematics \\ University of California \\ Los Angeles, CA 90095-1555 \\ popa@math.ucla.edu
}

\author{
Vyjayanthi Chari \\ Department of Mathematics \\ University of California \\ Riverside, CA 92521-0135 \\ chari@math.ucr.edu \\ Kefeng Liu \\ Department of Mathematics \\ University of California \\ Los Angeles, CA 90095-1555 \\ liu@math.ucla.edu \\ Jie Qing \\ Department of Mathematics \\ University of California \\ Santa Cruz, CA 95064 \\ qing@ cats.ucsc.edu
}

\section{PRODUCTION}

Silvio Levy, Scientific Editor, production@msp.org

\section{SUPPORTING INSTITUTIONS}

ACADEMIA SINICA, TAIPEI

CALIFORNIA INST. OF TECHNOLOGY

INST. DE MATEMÁTICA PURA E APLICADA

KEIO UNIVERSITY

MATH. SCIENCES RESEARCH INSTITUTE

NEW MEXICO STATE UNIV.

OREGON STATE UNIV.

\author{
STANFORD UNIVERSITY \\ UNIV. OF BRITISH COLUMBIA \\ UNIV. OF CALIFORNIA, BERKELEY \\ UNIV. OF CALIFORNIA, DAVIS \\ UNIV. OF CALIFORNIA, LOS ANGELES \\ UNIV. OF CALIFORNIA, RIVERSIDE \\ UNIV. OF CALIFORNIA, SAN DIEGO \\ UNIV. OF CALIF., SANTA BARBARA
}

\author{
Daryl Cooper \\ Department of Mathematics \\ University of California \\ Santa Barbara, CA 93106-3080 \\ cooper@math.ucsb.edu \\ Jiang-Hua Lu \\ Department of Mathematics \\ The University of Hong Kong \\ Pokfulam Rd., Hong Kong \\ jhlu@maths.hku.hk \\ Paul Yang \\ Department of Mathematics \\ Princeton University \\ Princeton NJ 08544-1000 \\ yang@math.princeton.edu
}

These supporting institutions contribute to the cost of publication of this Journal, but they are not owners or publishers and have no responsibility for its contents or policies.

See inside back cover or msp.org/pjm for submission instructions.

The subscription price for 2014 is US $\$ 410 /$ year for the electronic version, and \$535/year for print and electronic.

Subscriptions, requests for back issues and changes of subscribers address should be sent to Pacific Journal of Mathematics, P.O. Box 4163, Berkeley, CA 94704-0163, U.S.A. The Pacific Journal of Mathematics is indexed by Mathematical Reviews, Zentralblatt MATH, PASCAL CNRS Index, Referativnyi Zhurnal, Current Mathematical Publications and Web of Knowledge (Science Citation Index).

The Pacific Journal of Mathematics (ISSN 0030-8730) at the University of California, c/o Department of Mathematics, 798 Evans Hall \#3840, Berkeley, CA 94720-3840, is published twelve times a year. Periodical rate postage paid at Berkeley, CA 94704, and additional mailing offices. POSTMASTER: send address changes to Pacific Journal of Mathematics, P.O. Box 4163, Berkeley, CA 94704-0163.

PJM peer review and production are managed by EditFLOW ${ }^{\circledR}$ from Mathematical Sciences Publishers.

\section{PUBLISHED BY}

\section{mathematical sciences publishers \\ nonprofit scientific publishing}

http://msp.org/

(C) 2014 Mathematical Sciences Publishers 


\section{PACIFIC JOURNAL OF MATHEMATICS}

Volume 269 No. $1 \quad$ May 2014

The asymptotic behavior of Palais-Smale sequences on manifolds with $\quad 1$ boundary

SÉRGIO AlMARAZ

The cup subalgebra of a $\mathrm{II}_{1}$ factor given by a subfactor planar algebra is maximal amenable

ARNAUD BROTHIER

Representation theory of type B and C standard Levi $W$-algebras

JONATHAN BROWN and SIMON M. GOODWIN

New invariants for complex manifolds and rational singularities

RONG DU and YUN GAO

Homogeneity groups of ends of open 3-manifolds

DENNIS J. GARITY and DUŠAN REPOVŠ

On the concircular curvature of a $(\kappa, \mu, \nu)$-manifold

Florence Gouli-Andreou and Evaggelia Moutafi

Genuses of cluster quivers of finite mutation type

FANG LI, JICHUN LIU and YICHAO YANG

Taut foliations in knot complements

TAO LI and RACHEL ROBERTS

On the set of maximal nilpotent supports of supercuspidal representations 169

QIN YUJUN

The natural filtrations of finite-dimensional modular Lie superalgebras of 199 Witt and Hamiltonian type

Keli Zheng, Yongzheng ZHANG and WeI Song

Free Brownian motion and free convolution semigroups: multiplicative 219 case

PING ZHONG 